\title{
Grease matrix as a versatile carrier of proteins for serial crystallography.
}

\section{AUTHOR(S):}

Sugahara, Michihiro; Mizohata, Eiichi; Nango, Eriko; Suzuki, Mamoru; Tanaka, Tomoyuki; Masuda, Tetsuya; Tanaka, Rie; ... Joti, Yasumasa; Yabashi, Makina; Iwata, So

\section{CITATION:}

Sugahara, Michihiro ... [et al]. Grease matrix as a versatile carrier of proteins for serial crystallography.. Nature methods 2014, 12(1): 61-63

\section{ISSUE DATE:}

2014-11-10

URL:

http://hdl.handle.net/2433/203008

\section{RIGHT:}

This is the manuscript version of the article is available at

http://dx.doi.org/10.1038/nmeth.3172.; この論文は出版社版でありません。引用の際に は出版社版をご確認ご利用ください。; This is not the published version. Please cite only the published version. 


\title{
Grease matrix as a versatile carrier of proteins for serial crystallography
}

\author{
Michihiro Sugahara, ${ }^{1,}$ Eiichi Mizohata, ${ }^{2,}$ Eriko Nango, ${ }^{1,}$ Mamoru Suzuki, ${ }^{1,3,}$ Tomoyuki \\ Tanaka, ${ }^{1,}$ Tetsuya Masuda, ${ }^{1,4},{ }^{4}$ Rie Tanaka, ${ }^{1,}$ Tatsuro Shimamura, ${ }^{5}$, Yoshiki Tanaka, ${ }^{5}$, Chiyo \\ Suno, ${ }^{5}$ Kentaro Ihara, ${ }^{5,}$ Dongqing Pan, ${ }^{6}$, Keisuke Kakinouchi, ${ }^{7}$, Shigeru Sugiyama, ${ }^{7}$, \\ Michio Murata, ${ }^{7}$ Tsuyoshi Inoue,${ }^{2}$ Kensuke Tono, ${ }^{8,}$ Changyong Song ${ }^{1,}$ Jaehyun Park, ${ }^{1,}$ \\ Takashi Kameshima, ${ }^{8,}$ Takaki Hatsui, ${ }^{1,}$ Yasumasa Joti, ${ }^{8}$ Makina Yabashi ${ }^{1,}$ \& So Iwata ${ }^{1,5}$,
}

\section{Affiliations}

1. RIKEN SPring-8 Center, Sayo, Japan.

Michihiro Sugahara, Eriko Nango, Mamoru Suzuki, Tomoyuki Tanaka, Tetsuya Masuda, Rie Tanaka, Changyong Song, Jaehyun Park, Takaki Hatsui, Makina Yabashi \& So Iwata

2. Department of Applied Chemistry, Graduate School of Engineering, Osaka University, Suita, Japan.

Eiichi Mizohata \& Tsuyoshi Inoue

3. Laboratory of Supramolecular Crystallography, Research Center for Structural and Functional Proteomics, Institute for Protein Research, Osaka University, Suita, Japan.

Mamoru Suzuki

4. Division of Food Science and Biotechnology, Graduate School of Agriculture, Kyoto University, Uji, Japan.

Tetsuya Masuda

5. Department of Cell Biology, Graduate School of Medicine, Kyoto University, Sakyo, Japan.

Tatsuro Shimamura, Yoshiki Tanaka, Chiyo Suno, Kentaro Ihara \& So Iwata

6. Department of Structural Biology, Graduate School of Pharmaceutical Sciences, Kyoto University, Sakyo, Japan.

Dongqing Pan

7. Japan Science and Technology Agency (JST)-Exploratory Research for Advanced Technology (ERATO), Lipid Active Structure Project, Toyonaka, Japan.

Keisuke Kakinouchi, Shigeru Sugiyama \& Michio Murata

8. Japan Synchrotron Radiation Research Institute, Sayo, Japan.

Kensuke Tono, Takashi Kameshima \& Yasumasa Joti

Corresponding author

Correspondence to: Michihiro Sugahara (msuga@spring8.or.jp). 


\begin{abstract}
Serial femtosecond X-ray crystallography (SFX) has revolutionized atomic-resolution structural investigation by expanding applicability to micrometer-sized protein crystals, even at room temperature, and by enabling dynamics studies. However, reliable crystal-carrying media for SFX are lacking. Here we introduce a grease-matrix carrier for protein microcrystals and obtain the structures of lysozyme, glucose isomerase, thaumatin and fatty acid-binding protein type 3 under ambient conditions at a resolution of or finer than $2 \AA$.
\end{abstract}


Recent availability of intense, femtosecond X-ray pulses from X-ray free-electron lasers (XFELs) has opened a new era in structural biology by allowing the acquisition of diffraction patterns before the onset of X-ray radiation damage, or 'diffraction before destruction', via single-pulse exposures ${ }^{1-6}$. Diffraction signals up to a few angstroms in resolution can be obtained from even submicrometer-size crystals, thereby greatly alleviating the challenge of producing large crystals. A single-pulse X-ray exposure will completely destroy small individual crystals; therefore, fresh specimens must be supplied for subsequent X-ray pulses to continue data acquisition. This approach is known as SFX'. Since its first demonstration with photosystem I, SFX has been successfully applied to various protein systems ${ }^{7-10}$. In particular, the adaptability of SFX to proteins in different environments has invigorated structural analysis in vivo, at room temperature and during chemical reactions ${ }^{11,12}$.

SFX is contingent on a reliable and tractable supply of protein crystals to complete the data collection. Tens of thousands of diffraction patterns from specimens in random orientations are required to obtain a structure at a resolution of a few angstroms. One method of serial sample loading is to use liquid microjet injection ${ }^{13}$. With the continuous liquid flow forming a stable jet stream, a rather large sample is required, and less than $0.01 \%$ of the crystals are typically exposed to X-ray pulses ${ }^{14}$. This often requires $10-100 \mathrm{mg}$ of microcrystals; consequently, the applicability of SFX to proteins with low expression or poor crystallization is limited. Further, protein crystals stable in high-salt solutions or suspended in a viscous medium such as the lipidic cubic phase (LCP) require an alternative method. A microextrusion of crystals embedded in LCP directly from a syringe with a fine needle (inner diameter (i.d.) of $\sim 50 \mu \mathrm{m}$ ) enabled investigation of the $5-\mathrm{HT}_{2 \mathrm{~B}}$ receptor using much smaller sample amounts ( $\sim 0.3 \mathrm{mg})$, but this approach is probably limited to proteins crystallized in $\mathrm{LCP}^{15,16}$. A more universal method that is applicable to a wide variety of proteins is essential to firmly establish SFX. 
Here we introduce a mineral oil-based grease matrix as a generic carrier of protein microcrystals for SFX using XFELs. The grease matrix provides maximum adaptability for most classes of proteins with a straightforward sample-loading procedure, protection from structural deformation induced by the mechanical stress of osmotic pressure present in other viscous media, and preservation of the aqueous environment of the native protein molecules. We successfully applied the grease-matrix carrier to various proteins including lysozyme, glucose isomerase, thaumatin and fatty acid-binding protein type 3 (FABP3) in SFX experiments and obtained electron density maps beyond 2 - $\AA$ resolution using $<1 \mathrm{mg}$ of micrometer-sized protein crystals.

We carried out SFX experiments using femtosecond X-ray pulses from the SPring-8 Angstrom Compact Free Electron Laser (SACLA) . The X-ray wavelength was kept at $1.24 \AA$ (10 keV) with a pulse energy of $\sim 300 \mu \mathrm{J}$ contained for a 10-fs pulse duration in full-width at half-maximum (FWHM). We investigated four different types of protein crystals that are stable in different reagent solutions to demonstrate the general applicability of the grease matrix as a carrier (Supplementary Table 1). We suspended protein microcrystals in the grease medium with the following procedure (Supplementary Video 1). First, the microcrystal solution was dispensed into mineral oil-based grease spread on a glass slide by gentle mixing with a spatula (Fig. 1a). The grease matrix containing microcrystals was inserted into a dispenser tip (Fig. 1b). To keep the grease inside the tube, we sealed the dispenser tip with Parafilm. The dispenser tip was centrifuged for a few seconds, and an aliquot of the sample was loaded into a syringe (Fig. 1c). We used storage solutions of protein microcrystals: lysozyme (7-10 $\mu \mathrm{m}$ in maximum length), glucose isomerase (10-30 $\mu \mathrm{m})$, thaumatin $(10-30 \mu \mathrm{m})$ and FABP3 $(10-20 \mu \mathrm{m})$ (Supplementary Fig. 1). For SFX data collection, we used a sample volume of $15-27 \mu \mathrm{l}\left(10^{7}\right.$ crystals $\left./ \mathrm{ml}\right)$. The grease matrix containing randomly oriented protein microcrystals was continuously delivered to the $1.5-\mu \mathrm{m}$ (FWHM)-diameter focal spot of the XFEL radiation using a syringe injector with a $110-\mu m$-i.d. 
needle. A flow rate of $0.48 \mu \mathrm{min}^{-1}$ was used for lysozyme, glucose isomerase and FABP3, and $0.46 \mu \mathrm{l} \mathrm{min}{ }^{-1}$ was used for thaumatin. The grease formed a stable flow for all protein samples (Fig. 1d,e).

We collected single-pulse X-ray diffraction patterns of microcrystals from the four different proteins. With the SACLA running at a $30-\mathrm{Hz}$ repetition rate, $\sim 100,000$ diffraction patterns were collected within $1 \mathrm{~h}$ using less than $30 \mu \mathrm{l}$ of the sample volume (Supplementary Table 2). We successfully indexed and integrated 8,000-30,000 patterns for each of the proteins. Lysozyme, glucose isomerase, thaumatin and FABP3 crystals belong to space groups $P 4_{3} 2_{1} 2, I 222, P 4_{1} 2_{1} 2$ and $P 2_{1} 2_{1} 2_{1}$, respectively; the crystals yielded data sets beyond $2.0-\AA$ resolution with a completeness of $99.9-100 \%$ and an $R_{\text {split }}$ (ref. 17) ranging from $8.7 \%$ to $28.9 \%$. We think that the large variation in the $R_{\text {split }}$ values may reflect the lack of uniformity of crystals used in this study (Supplementary Fig. 1). We determined the room-temperature crystal structures of lysozyme (Protein Data Bank ID: 3WUL), glucose isomerase (4W4Q), thaumatin (3WXS) and FABP3 (3WXQ) at 2.0, 2.0, 2.0 and $1.6 \AA$ and refined to an $R / R_{\text {free }}$ of $20.7 / 23.0 \%, 15.9 / 19.6 \%, 15.7 / 20.0 \%$ and $17.8 / 22.2 \%$, respectively. We were able to observe clear electron density maps; examples are shown here for lysozyme and glucose isomerase (Fig. 2 and Supplementary Fig. 2).

In SFX experiments, a slower flow rate can reduce sample consumption. To demonstrate the viability of a grease-matrix carrier for slow flow injection, we performed experiments at two different flow rates, 0.24 and $0.12 \mu \mathrm{min}^{-1}$, using lysozyme crystals injected through a $110-\mu m$-i.d. needle. There was no noticeable difference in the indexing rates or in the diffraction pattern obtained at 2-Å resolution (Supplementary Table. 2), implying that a flow rate as low as $0.12 \mu \mathrm{min}^{-1}$ is sufficient for the $30-\mathrm{Hz}$ operation. To further reduce sample consumption, we tested a syringe injector using a 50- $\mu m$-i.d. needle with a grease matrix containing 7- to 10- $\mu \mathrm{m}$ lysozyme crystals. Under the same experimental 
conditions, we obtained a diffraction data set with $99.9 \%$ completeness at a resolution of 2.0 $\AA$ (Supplementary Table 2). The indexing rate was $29 \%$, similar to that using the $110-\mu \mathrm{m}$ i.d. needle. From $\sim 21 \mu$ of lysozyme crystals dispersed in grease, we recorded a total of

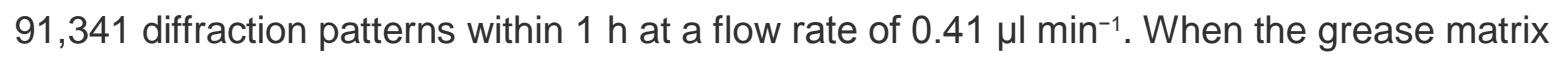
was passed smoothly through the $50-\mu m$-i.d. needle at a reduced flow rate, we substantially lowered sample consumption, using only $\sim 0.1 \mathrm{mg}$ of the sample instead of $\sim 1 \mathrm{mg}$ with a 110 $\mu m$-i.d. needle. The mineral oil-based grease extruded through the $110-\mu m-i . d$. needle tended to produce a larger-diameter grease column (approximately $210-240 \mu \mathrm{m}$ ) similar to the outer diameter (o.d.) of the needle (Fig. 1e). We therefore suggest using needles with smaller o.d. to reduce the diameter of the grease column.

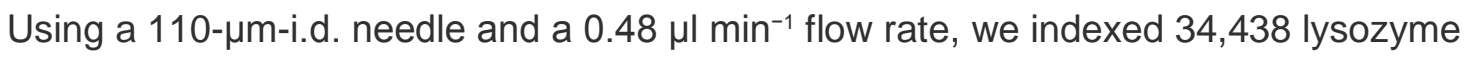
patterns from the total of 101,330 collected to give a 34\% index rate (Supplementary Table 2). Under similar conditions, we obtained index rates of $15.2 \%, 10.5 \%$ and $24.4 \%$ from glucose isomerase, thaumatin and FABP3, respectively. An increase in the hit rate can be accommodated with increased crystal number densities, which would, however, cause multiple sample hits in one shot and complicate the indexing procedures. We found that a crystal number density of $\sim 10^{7}$ was suitable for SFX data collection under our experimental conditions using the $110-\mu m-i . d$. needle, which was consistent with the results from a previous simulation study ${ }^{14}$.

In summary, using a mineral oil-based grease matrix as a general carrier of protein microcrystals for serial sample loading in SFX, we successfully obtained room-temperature structures beyond $2-\AA \AA$ resolution from four different types of proteins in $\sim 7$ - to $30-\mu \mathrm{m}$ microcrystals using $<1 \mathrm{mg}$ of sample. Larger crystals are vulnerable to repeated exposures to $\mathrm{X}$-ray pulses and may degrade from the radiation damage; microcrystals may escape this fate, but it is not completely ruled out in this study. Use of a grease-matrix carrier for proteins 
may expand SFX application to environmentally hazardous proteins-for example, heavyatom replacements such as $\mathrm{Hg}$ —by virtue of the controlled collection of the grease medium during the experiment. Furthermore, the grease-matrix carrier of microcrystals should be applicable for high-repetition XFELs of Euro-XFEL $(\sim 30 \mathrm{kHz})$ currently under construction and LCLS-II (>100 kHz) on the planned upgrade to streamline data collections. This, however, will be contingent on technical improvements of the syringe system to accommodate an injection rate $2-3$ orders of magnitude higher than it is now. Our grease matrix-based approach should be applicable to a wide range of proteins as well as inorganic nanocrystals to facilitate investigations in atomic-resolution structure determination using single-pulse exposures.

\section{Online Methods}

\section{Sample preparation.}

Crystallization of lysozyme for the grease technique was performed by mixing a 1:1 ratio of $20 \mathrm{mg} \mathrm{ml}^{-1}$ protein solution in $0.1 \mathrm{M}$ sodium acetate $(\mathrm{pH} 3.0)$ and precipitant solution composed of 28\% (w/v) sodium chloride, 8\% (w/v) PEG6000 and $0.1 \mathrm{M}$ sodium acetate (pH 3.0) $)^{7,18}$. Crystals with a uniform size distribution of 7-10 $\mu \mathrm{m}$ appeared after incubation at $20^{\circ} \mathrm{C}$. A 40-ml sample of crystallization solution was centrifuged at $4{ }^{\circ} \mathrm{C}$ and $3,000 \mathrm{~g}$ for 3 min. After the supernatant solution was completely removed by decantation, crystals were suspended in $10 \mathrm{ml}$ of $10 \%(\mathrm{w} / \mathrm{v})$ sodium chloride and $1.0 \mathrm{M}$ sodium acetate $(\mathrm{pH} 3.0)$.

Glucose isomerase was crystalized by mixing a 1:1 ratio of $83 \mathrm{mg} \mathrm{ml}^{-1}$ protein solution in $10 \mathrm{mM}$ HEPES (pH 7.0) and precipitant solution composed of 40\% (w/v) PEG4000, $0.2 \mathrm{M}$ lithium sulfate and 0.1 M Tris- $\mathrm{HCl}(\mathrm{pH} 8.5)$ on ice. Seed crystals were added to the solution, and microcrystals were produced by incubation for $1 \mathrm{~d}$ at $5^{\circ} \mathrm{C}$. A 1.0-ml sample of crystallization solution was centrifuged at $4{ }^{\circ} \mathrm{C}$ and $3,000 \mathrm{~g}$ for $3 \mathrm{~min}$, and then the supernatant solution was removed by decantation. The crystals of glucose isomerase were 
suspended in $1.0 \mathrm{ml}$ of 30\% (w/v) PEG4000, $0.2 \mathrm{M}$ lithium sulfate and $0.1 \mathrm{M}$ Tris- $\mathrm{HCl}(\mathrm{pH}$ 8.5).

Thaumatin I was purified from crude thaumatin powder by ion-exchange column chromatography followed by gel filtration as described previously ${ }^{19}$. The purified thaumatin I was concentrated by a Centricon 10 (Millipore), and the protein concentration was measured with a NanoDrop ND-1000 spectrophotometer (NanoDrop Technologies). Crystallization was performed by using the batch method. Microcrystals were obtained by mixing an equal volume of the $40 \mathrm{mg} \mathrm{ml}^{-1}$ protein solutions and the reservoir solution, which consisted of 0.1 M ADA and 2.0 M potassium sodium tartrate ( $\mathrm{pH}$ 7.2). After centrifugation, crystals were suspended in $0.1 \mathrm{M}$ ADA and $0.75 \mathrm{M}$ potassium sodium tartrate $(\mathrm{pH} 7.0)$.

FABP3 was purified as described previously ${ }^{20}$. The purified protein was mixed with stearic acid (C18:0), and the protein concentration was adjusted to $20.5 \mathrm{mg} \mathrm{ml}^{-1}$ in $20 \mathrm{mM}$ Tris- $\mathrm{HCl}(\mathrm{pH}$ 8.0) buffer containing $100 \mathrm{mM} \mathrm{NaCl}$. Crystallization was performed by the batch method in a 15-ml centrifuge tube (AS ONE Co.). Microcrystals were obtained by mixing 2.83 $\mathrm{ml}$ of the protein solution with $5.67 \mathrm{ml}$ of precipitant solution, which was composed of 100 $\mathrm{mM}$ Tris- $\mathrm{HCl}(\mathrm{pH} 8.0)$ and 65\% (v/v) PEG400. The microseeding technique promoted the appropriate size of crystal formation within $1 \mathrm{~d}$.

Details on crystal size, crystal number density and storage solutions specific to each protein are summarized in Supplementary Table 1. Crystals were suspended in their own storage solutions. The crystal suspensions were filtered through a mesh (pore size, $30 \mu \mathrm{m}$ ) and adjusted to a number density of approximately $10^{7}$ crystals $/ \mathrm{ml}$. All samples were stored at $4{ }^{\circ} \mathrm{C}$. Protein microcrystals were prepared following procedures detailed below (Supplementary Video 1). After a 100- $\mu$ l sample of storage solution was centrifuged for 10

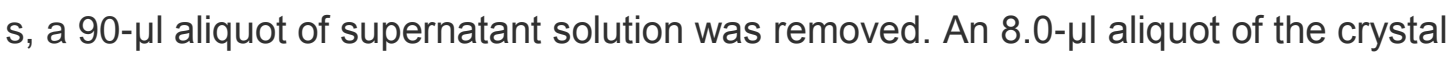
solution was dispensed into $72 \mu \mathrm{l}$ of mineral oil-based grease (\#761, AZ Co., 
https://www.az-oil.jp/) on a glass slide and then mixed (Fig. 1a). The synthetic grease Super Lube (\#21030, Synco Chemical Co., http://www.super-lube.com/) is a good alternative to the AZ grease. The crystal-containing grease was inserted into a dispenser tip using a spatula (Fig. 1b). The sample was concentrated in the top of the dispenser tip by centrifuging the tip for $2-3 \mathrm{~s}$. An aliquot $(30 \mu \mathrm{l})$ of the sample was extruded into a $100-\mu \mathrm{l}$ syringe (\#1710, Hamilton) by applying pressure from the wider end of the dispenser tip using a 1.0-ml disposable syringe (SS-01T, Terumo, Fig. 1c).

\section{Single-shot data collection.}

The syringe injector system was installed in a helium ambiance, diffraction chamber enclosure: Diverse Application Platform for Hard X-ray Diffraction in SACLA (DAPHNIS). To control the sample flow speed from the syringe, a stepper motor drove the syringe plunger. The sample holder was then exposed to a cooled helium gas stream that passed through a liquid nitrogen trap. The microcrystals embedded in grease were kept at a temperature of 19.5-21. $7^{\circ} \mathrm{C}$. The sample chamber was kept at a temperature of $21.3-23.6^{\circ} \mathrm{C}$ and a humidity of 2-9\%. Diffraction patterns were collected using a short-working-distance octal multiport CCD detector ${ }^{21}$ with XFEL radiation at BL3 of SACLA, Japan ${ }^{22}$. The microcrystals were exposed to single X-ray pulses at a photon energy of $10 \mathrm{keV}$. Pulses, which were of 10 fs duration, consisted of $7 \times 10^{10}$ photons per pulse focused to $1.5 \times 1.5 \mu \mathrm{m}^{2}$ at the interaction point using Kirkpatrick-Baez mirrors ${ }^{23}$. The repetition rate was $30 \mathrm{~Hz}$, and the average 10-fs pulse energy at the sample was $120 \mu \mathrm{J}$ per pulse. The mineral oil-based grease with randomly oriented crystals was extruded through two types of syringe needles with an inner diameter (i.d.) of $110 \mu \mathrm{m}$ (outer diameter (o.d.), $240 \mu \mathrm{m}$; \#7803-04, Hamilton) or $50 \mu \mathrm{m}$ (o.d., $160 \mu \mathrm{m}$; \#207434, Hamilton). The sample flow rate was $0.48 \mu \mathrm{l} \mathrm{min}^{-1}$ for the $110-\mu \mathrm{m}$ i.d. needle. In order to reduce the rate of sample consumption, the lysozyme crystal- 
containing grease was extruded at a constant flow rate of 0.24 or $0.12 \mu \mathrm{l} \mathrm{min}{ }^{-1}$ from the 110 $\mu m-i . d$. needle. In addition, lysozyme crystals dispersed in the grease were extruded through

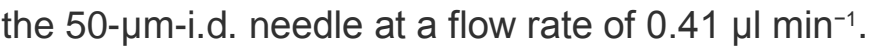

\section{Structure determination.}

Diffraction patterns were indexed and integrated using CrystFEL ${ }^{17}$. Diffraction peak positions were determined using the built-in Zaefferer algorithm and passed on to DirAx ${ }^{24}$ and MOSFLM ${ }^{25,26}$ for indexing. After Monte Carlo integrated intensities from CrystFEL were converted to XDS format, the intensities were also converted to MTZ format using XDSConv ${ }^{27,}{ }^{28}$. The structures were determined by the molecular replacement method using Phaser ${ }^{29}$ with search models (PDB: 1VDS for lysozyme, 1MNZ for glucose isomerase, 3AL7 for thaumatin and 3WVM for FABP3). Manual model revision was performed using Quanta2000 (Accelrys) and Coot $^{30}$ softwares. The programs Refmac from the CCP4 suite ${ }^{31}$ and PHENIX ${ }^{32}$ were used for structure refinement. Results of data collection and refinement statistics are summarized in Supplementary Table 2.

\section{Additional information}

\section{Accession codes.}

Protein Data Bank: Coordinates and structure factors have been deposited under accession codes $\mathbf{3 W U L}$, 3WXT, 3WXU and 3WUM (lysozyme); 4W4Q (glucose isomerase); 3WXS (thaumatin); and 3WXQ (FABP3).

\section{Competing financial interests}

The authors declare no competing financial interests. 


\section{Acknowledgments}

The XFEL experiments were carried out at the BL3 of SACLA with the approval of the Japan Synchrotron Radiation Research Institute (JASRI) (proposal nos. 2012B8036, 2013A8039, 2013A8040, 2013B8044, 2013B8045 and 2014A8032). This work was supported by RIKEN, by the X-ray Free-Electron Laser Priority Strategy Program (MEXT) and partly by Research Acceleration Program of Japan Science and Technology Agency. The sample preparation of FABP3 was supported by the JST-ERATO Murata Lipid Active Structure Project. The authors thank the SACLA beamline staff for technical assistance, $\mathrm{K}$. Diederichs for help with data analysis and A. Nisbet for careful reading of the manuscript.

\section{Contributions}

M. Sugahara introduced grease-matrix extrusion scheme. M. Sugahara, E.M., E.N., M. Suzuki, T.T. and T.M. performed data collection, data processing, structure refinements (lysozyme: M. Sugahara and E.N.; glucose isomerase: E.N. and T.T.; thaumatin: T.M.; FABP3: E.M. and M. Suzuki). E.M., E.N., T.T., T.M., T.S., Y.T., C. Suno, K.I., D.P., K.K., S.S., M.M. and T.I. developed the microcrystal sample preparations and prepared samples (lysozyme: T.S., Y.T., C. Suno, K.I., D.P., E.N. and T.T.; glucose isomerase: E.N. and T.T.; thaumatin: T.M.; FABP3: E.M., K.K., S.S., M.M. and T.I.). E.N. and R.T. designed and developed the injection method. K.T. and M.Y. developed the DAPHNIS. K.T., C. Song, J.P., T.K., T.H., Y.J. and M.Y. developed the SFX systems including injectors. M. Sugahara, E.N. and C. Song wrote the manuscript with input from all the coauthors. S.I. coordinated the project. 


\section{References}

1. Schlichting, I. \& Miao, J. Curr. Opin. Struct. Biol. 22, 613-626 (2012).

2. Chapman, H.N. et al. Nature 470, 73-77 (2011).

3. Neutze, R., Wouts, R., van der Spoel, D., Weckert, E. \& Hajdu, J. Nature 406, 752-757 (2000).

4. Barty, A. et al. Nat. Photonics 6, 35-40 (2012).

5. Emma, P. et al. Nat. Photonics 4, 641-647 (2010).

6. Ishikawa, T. et al. Nat. Photonics 6, 540-544 (2012).

7. Boutet, S. et al. Science 337, 362-364 (2012).

8. Barends, T.R.M. Nature 505, 244-247 (2014).

9. Johansson, L.C. et al. Nat. Methods 9, 263-265 (2012).

10. Redecke, L. et al. Science 339, 227-230 (2013).

11. Koopmann, R. et al. Nat. Methods 9, 259-262 (2012).

12. Kern, J. et al. Science 340, 491-495 (2013).

13. Weierstall, U., Spence, J.C.H. \& Doak, R.B. Rev. Sci. Instrum. 83, 035108 (2012).

14. Park, J., Joti, Y., Ishikawa, T. \& Song, C. Appl. Phys. Lett. 103, 264101 (2013).

15. Weierstall, U. et al. Nat. Commun. 5, 3309 (2014). 
16. Liu, W. et al. Science 342, 1521-1524 (2013).

17. White, T.A. et al. J. Appl. Cryst. 45, 335-341 (2012).

18. Falkner, J.C. et al. Chem. Mater. 17, 2679-2686 (2005).

19. Masuda, T., Ohta, K., Mikami, B. \& Kitabatake, N. Acta Crystallogr. F Struct. Biol. Commun. 67, 652-658 (2011).

20. Hirose, M. et al. J. Synchrotron Radiat. 20, 923-928 (2013).

21. Kameshima, T. et al. Rev. Sci. Instrum. 85, 033110 (2014).

22. Tono, K. et al. New J. Phys. 15, 083035 (2013).

23. Yumoto, H. et al. Nat. Photonics 7, 43-47 (2013).

24. Duisenberg, A.J.M. J. Appl. Cryst. 25, 92-96 (1992).

25. Leslie, A.G.W. Acta Crystallogr. D Biol. Crystallogr. 62, 48-57 (2006).

26. Powell, H.R. Acta Crystallogr. D Biol. Crystallogr. 55, 1690-1695 (1999).

27. Kabsch, W. J. Appl. Cryst. 26, 795-800 (1993).

28. Kabsch, W. Acta Crystallogr. D Biol. Crystallogr. 66, 125-132 (2010).

29. McCoy, A.J. et al. J. Appl. Cryst. 40, 658-674 (2007).

30. Emsley, P. \& Cowtan, K. Acta Crystallogr. D Biol. Crystallogr. 60, 2126-2132 (2004).

31. Collaborative Computational Project, Number 4. Acta Crystallogr. D Biol. Crystallogr. 50, 760-763 (1994). 
32. Adams, P.D. et al. Acta Crystallogr. D Biol. Crystallogr. 58, 1948-1954 (2002). 


\section{Figure legends}

Figure 1 | Grease-matrix carrier of protein microcrystals and its extrusion. (a) Protein crystals and mineral oil-based grease were mixed on a plate. (b) The mixture was inserted into a dispenser tip. (c) The sample was loaded into a syringe. (d) A syringe needle was used to extrude the microcrystal-containing grease. (e) Grease was extruded as a continuous column to intersect with the XFEL beam.

Figure 2 | Electron density map of lysozyme obtained using microcrystals embedded in grease. Grease was extruded through a $110-\mu \mathrm{m}$-i.d. needle at a flow rate of $0.48 \mu \mathrm{min}^{-1}$. (a) Typical XFEL single diffraction pattern from an individual lysozyme microcrystal in grease. Resolution at the edges corresponds to $\sim 1.6-\AA$. The grease tended to give a diffraction ring pattern at $\sim 14-\AA \AA$ resolution and background diffraction in the resolution range of $4-5 \AA$. (b) Close-up view of the lysozyme structure (PDB: 3WUL) with 2Fo - FC electron density map contoured at the $1.0 \sigma$ level. This figure was drawn with PyMol. 
Figure 1: Grease-matrix carrier of protein microcrystals and its extrusion.
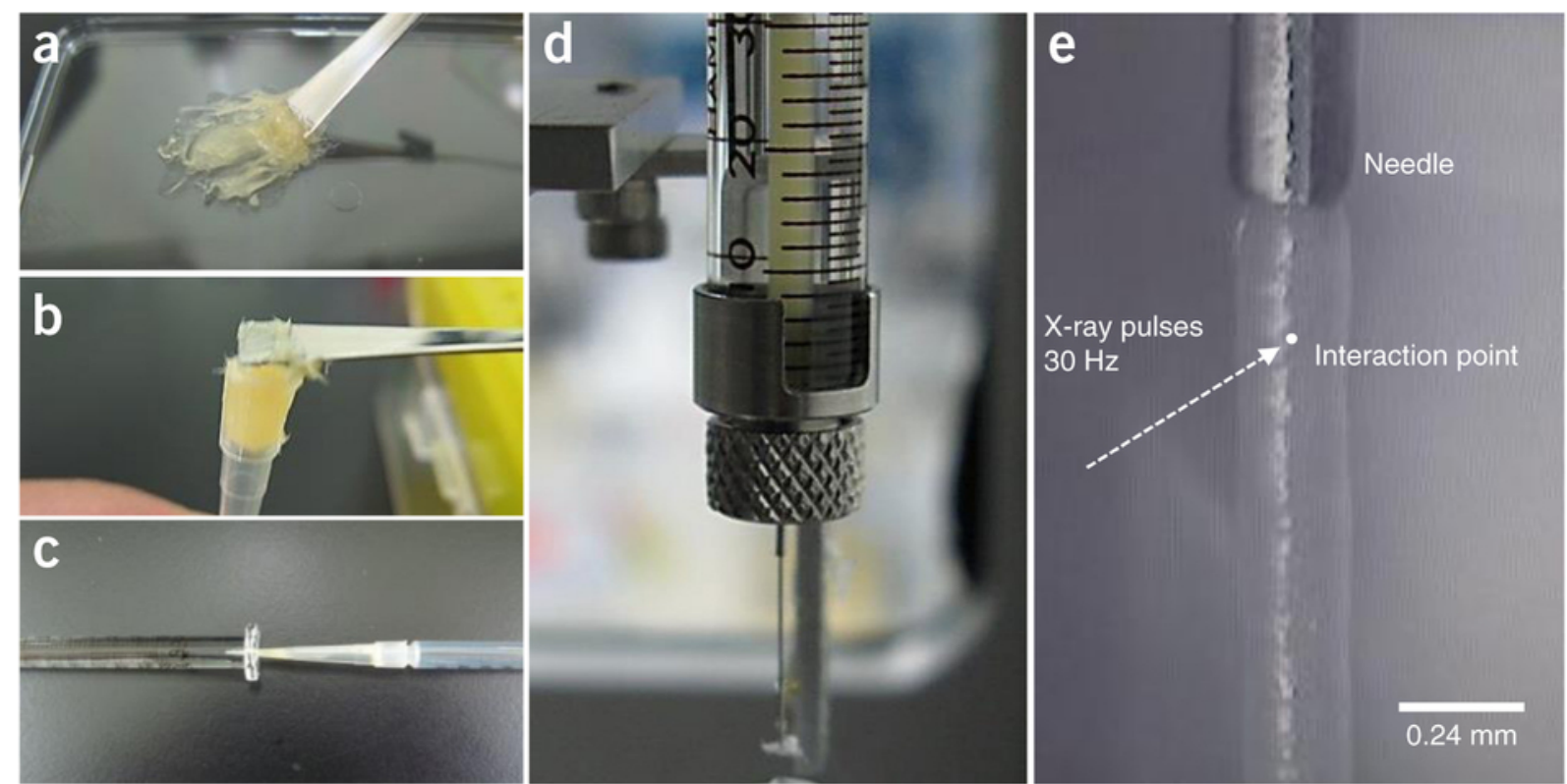
Figure 2: Electron density map of lysozyme obtained using microcrystals embedded in grease.
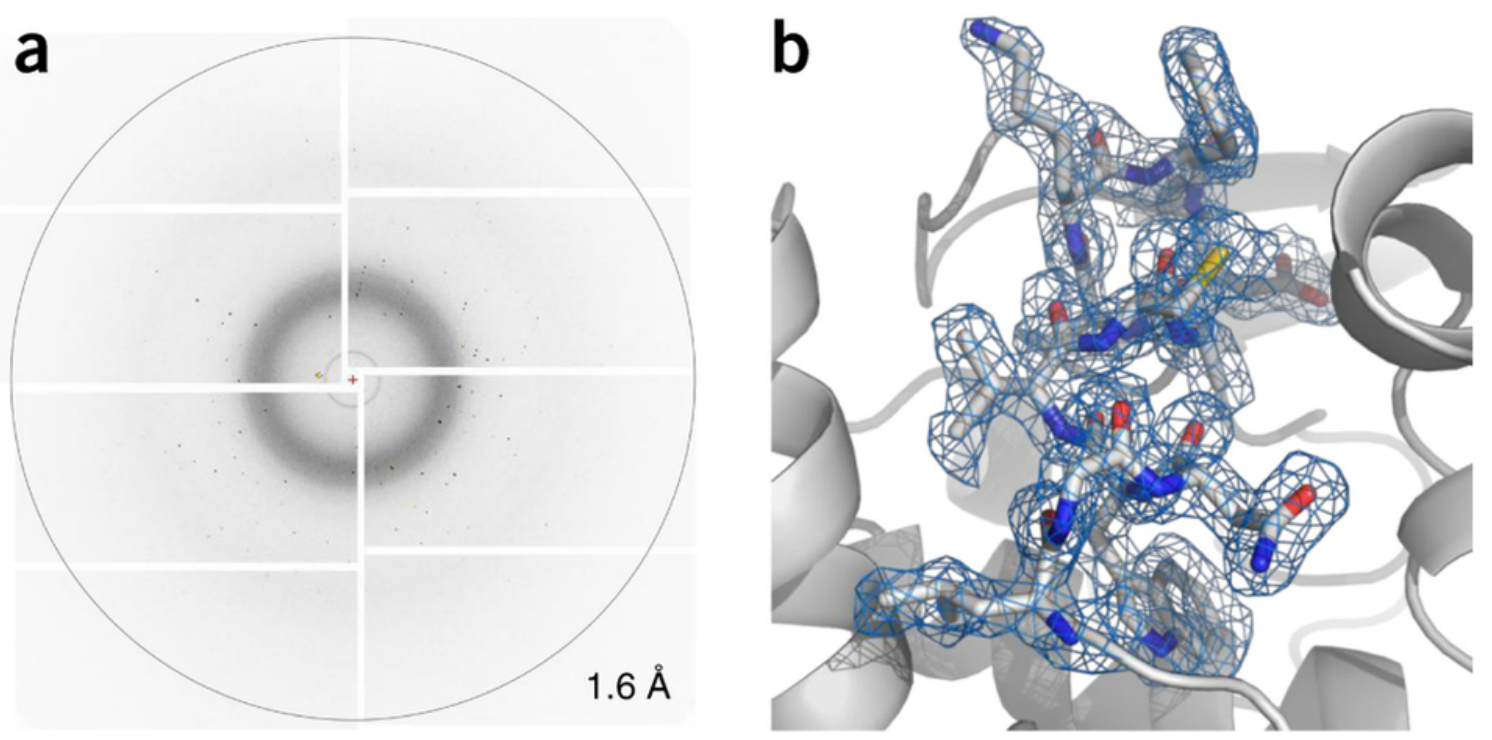
Supplementary Figure 1: Protein microcrystals used for SFX measurements.

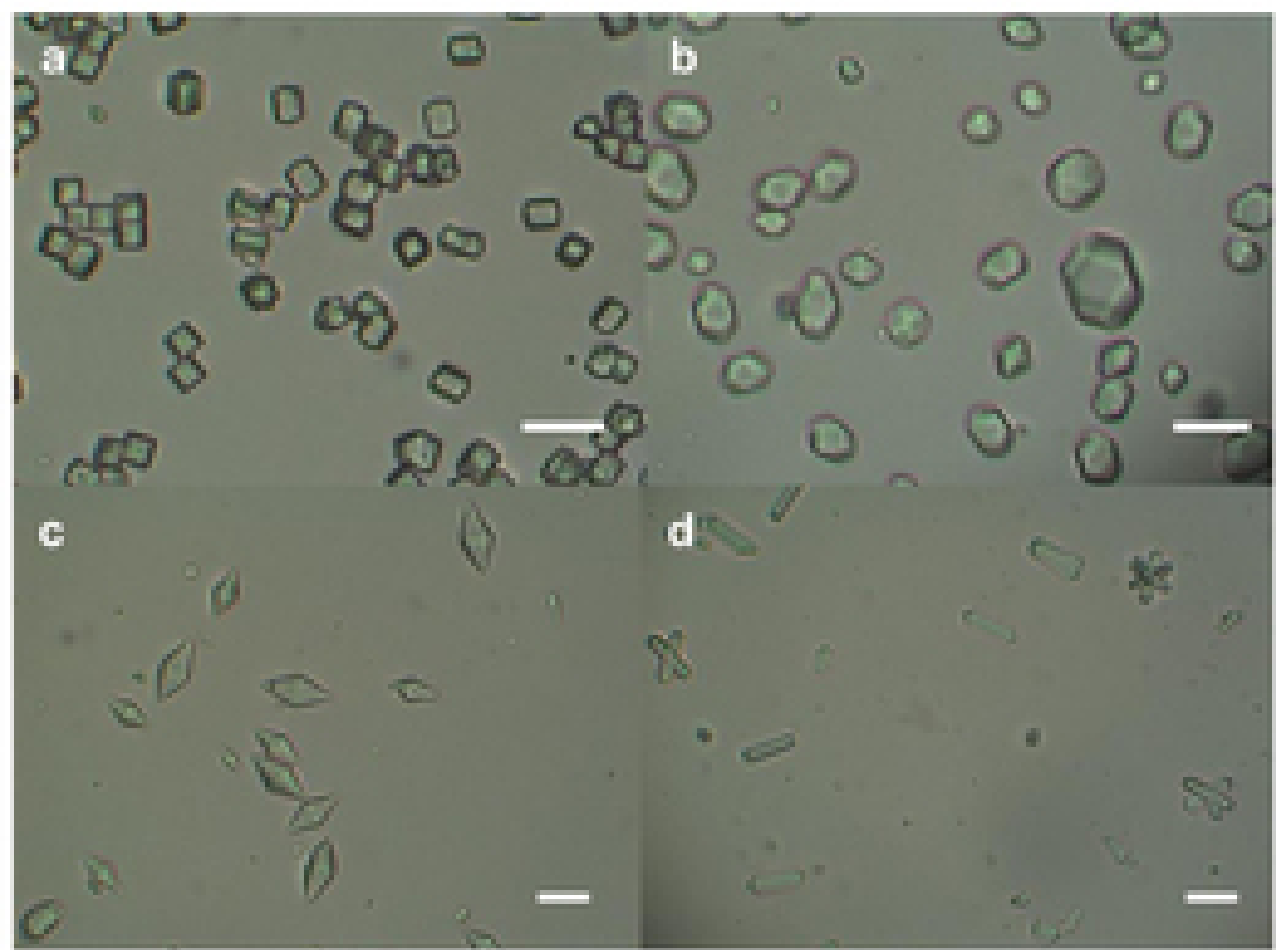

(a) lysozyme, (b) glucose isomerase, (c) thaumatin and (d) FABP3 crystals. Scale bars represent $20 \mu \mathrm{m}$. 
Supplementary Figure 2: Room-temperature structure of glucose isomerase.
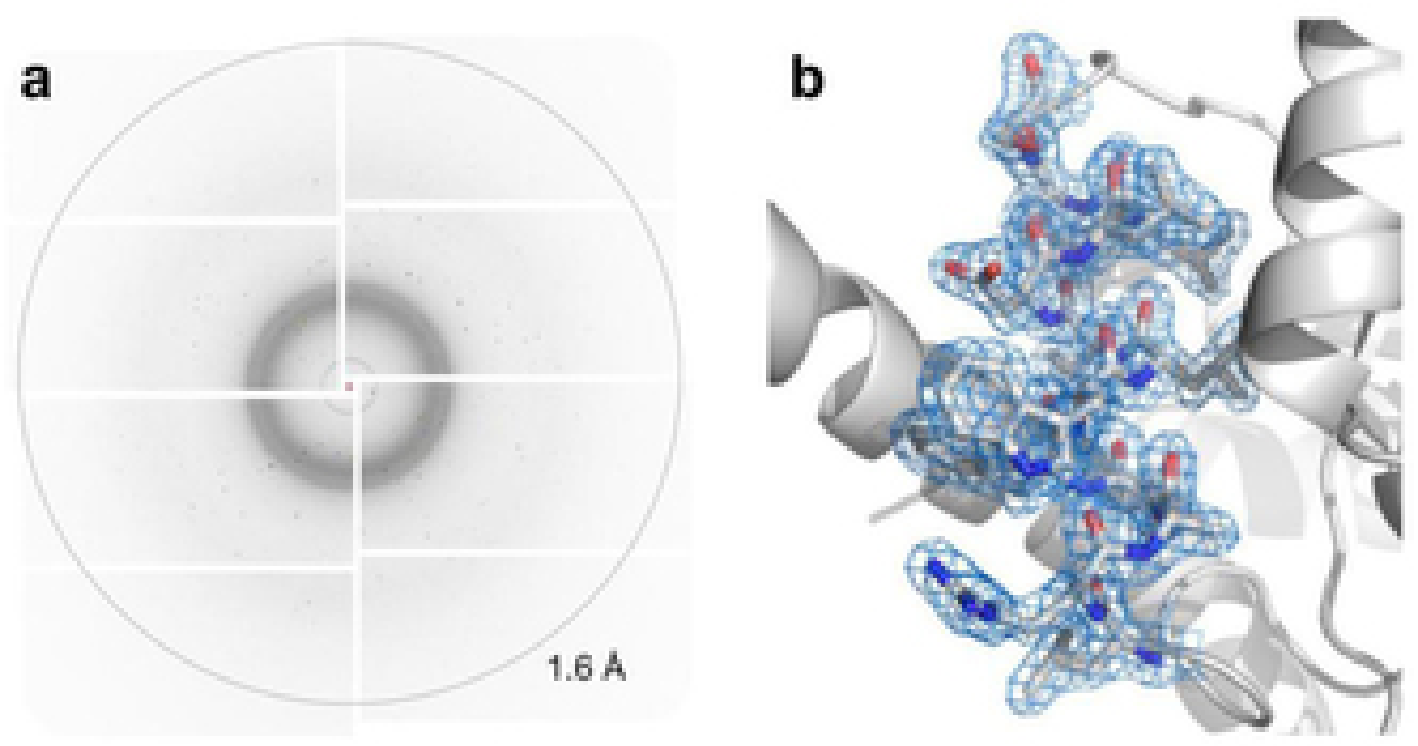

(a) A typical diffraction pattern from an individual microcrystal. Resolution at the edges corresponds to $\sim 1.6 \AA$. (b) A close-up view of glucose isomerase structure with $(2 F o-F C$ ) electron-density map (contoured at $1.0 \sigma)$. This figure was drawn with the program PyMol (http://www.pymol.org). 
Supplementary Table 1. Summary of crystal size, crystal number density and storage solutions for four proteins.

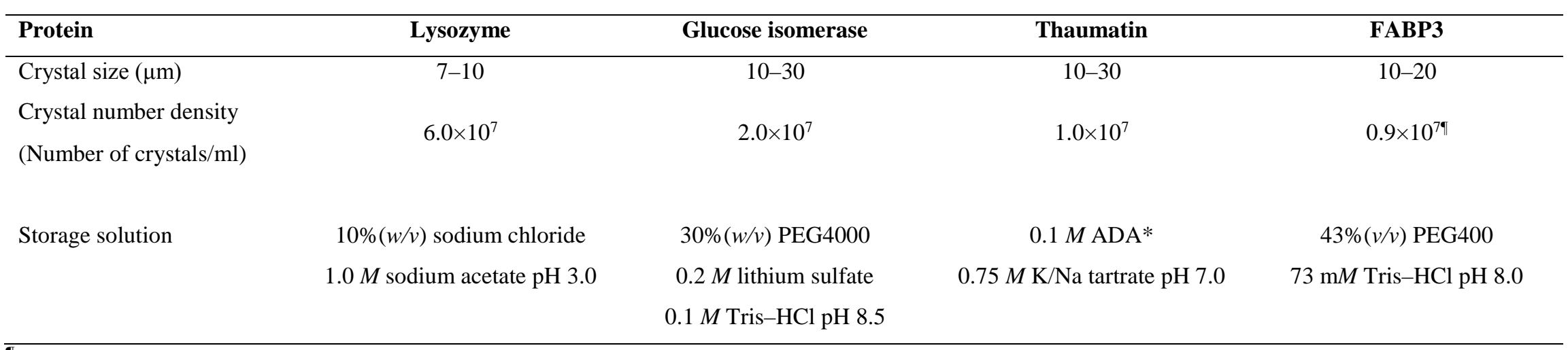

"About 30\% of this includes crystals formed in cluster.

*ADA: N-(2-acetamido) iminodiacetic acid 
Supplementary Table 2. Crystallographic statistics. Values in parentheses are for the outermost shell.

\begin{tabular}{|c|c|c|c|c|}
\hline Protein & Lysozyme & Lysozyme & Lysozyme & Lysozyme \\
\hline Needle ID $(\mu \mathrm{m})$ & 110 & 110 & 110 & 50 \\
\hline Flow rate $\left(\mu \mathrm{l} \mathrm{min}{ }^{-1}\right)$ & 0.48 & 0.24 & 0.12 & 0.41 \\
\hline \multicolumn{5}{|l|}{ Data collection } \\
\hline Space group & $P 4_{3} 2_{1} 2$ & $P 4_{3} 2_{1} 2$ & $P 4_{3} 2_{1} 2$ & $P 4_{3} 2_{1} 2$ \\
\hline \multicolumn{5}{|l|}{ Unit-cell parameter } \\
\hline$a(\AA)$ & 79 & 79 & 79 & 79 \\
\hline$b(\AA)$ & 79 & 79 & 79 & 79 \\
\hline$c(\AA)$ & 38 & 38 & 38 & 38 \\
\hline Number of collected images & 101,330 & 48,743 & 86,305 & 91,341 \\
\hline Number of indexed patterns & 34,438 & 14,595 & 29,323 & 26,646 \\
\hline Indexing rate $(\%)^{\S}$ & 34.0 & 29.9 & 34.0 & 29.2 \\
\hline Consumed protein per 1,000 indexed patterns ( $\mu \mathrm{g}$ ) & 31 & 18 & 8 & 31 \\
\hline Number of total reflections & $4,566,054$ & $1,950,298$ & 3,932,325 & $3,508,692$ \\
\hline Number of unique reflections & 8,548 & 8,549 & 8,548 & 8,548 \\
\hline Resolution range $(\AA)$ & $30-2.0(2.04-2.00)$ & $30-2.0(2.04-2.00)$ & $30-2.0(2.04-2.00)$ & $30-2.0(2.04-2.00)$ \\
\hline Completeness (\%) & $99.9(100)$ & $99.9(100)$ & $99.9(100)$ & $99.9(100)$ \\
\hline$R_{\text {split }}(\%)^{\dagger}$ & $8.7(8.3)$ & $12.7(15.3)$ & $7.7(9.9)$ & $9.5(8.6)$ \\
\hline $\mathrm{CC}_{1 / 2}(\%)$ & 97.8 (98.7) & $96.3(96.5)$ & 98.7 (98.1) & $97.1(97.9)$ \\
\hline$<I / \sigma(I)>$ & $12.4(10.8)$ & $7.9(5.9)$ & $13.3(8.8)$ & $11.8(11.4)$ \\
\hline \multicolumn{5}{|l|}{ Refinement } \\
\hline$R / R_{\text {free }}(\%)$ & $20.7 / 23.0$ & 19.8/23.0 & $18.5 / 21.1$ & 21.8/22.6 \\
\hline \multicolumn{5}{|l|}{ R.m.s. deviations } \\
\hline Bond lengths ( $\AA$ ) & 0.022 & 0.021 & 0.023 & 0.022 \\
\hline Bond angles $\left({ }^{\circ}\right)$ & 1.512 & 1.557 & 1.762 & 1.574 \\
\hline PDB code & 3wul & 3wxt & $3 w x u$ & 3wum \\
\hline
\end{tabular}

$\S$ Percentage of images that were indexed.

${ }^{\dagger} R_{\text {split }}=1 / \sqrt{2} \frac{\sum_{h k l}\left|I_{\text {even }}-I_{\text {odd }}\right|}{1 / 2 \sum_{h k l}\left|I_{\text {even }}+I_{\text {odd }}\right|}$ 


\begin{tabular}{|c|c|c|c|}
\hline Protein & Glucose isomerase & Thaumatin & FABP3 \\
\hline Needle ID $(\mu \mathrm{m})$ & 110 & 110 & 110 \\
\hline Flow rate $\left(\mu l \mathrm{~min}^{-1}\right)$ & 0.48 & 0.46 & 0.48 \\
\hline \multicolumn{4}{|l|}{ Data collection } \\
\hline Space group & I222 & $P 4_{1} 2_{1} 2$ & $P 2_{1} 2_{1} 2_{1}$ \\
\hline \multicolumn{4}{|l|}{ Unit-cell parameter } \\
\hline$a(\AA)$ & 94 & 58 & 34 \\
\hline$b(\AA)$ & 100 & 58 & 55 \\
\hline$c(\AA)$ & 103 & 150 & 71 \\
\hline Number of collected images & 67,792 & 84,078 & 54,256 \\
\hline Number of indexed patterns & 10,297 & 8,856 & 13,231 \\
\hline Indexing rate (\%) & 15.2 & 10.5 & 24.4 \\
\hline Consumed protein per 1,000 indexed patterns $(\mu \mathrm{g})$ & 73 & 32 & 11 \\
\hline Number of total reflections & $2,817,002$ & $2,415,636$ & $2,147,575$ \\
\hline Number of unique reflections & 33,122 & 18,071 & 18,204 \\
\hline Resolution range $(\AA)$ & $30-2.0(2.04-2.00)$ & $30-2.0(2.04-2.00)$ & $30-1.60(1.63-1.60)$ \\
\hline Completeness (\%) & $99.9(100)$ & $99.9(99.9)$ & $100(100)$ \\
\hline$R_{\text {split }}(\%)^{\dagger}$ & $28.9(33.9)$ & $16.4(15.9)$ & $20.0(38.2)$ \\
\hline $\mathrm{CC}_{1 / 2}(\%)$ & $85.2(75.5)$ & $91.8(94.9)$ & $91.5(79.9)$ \\
\hline$<I / \sigma(I)>$ & $3.60(3.0)$ & $6.4(5.6)$ & $4.9(2.5)$ \\
\hline \multicolumn{4}{|l|}{ Refinement } \\
\hline$R / R_{\text {free }}$ & 15.9/19.6 & $15.7 / 20.0$ & $17.8 / 22.2$ \\
\hline \multicolumn{4}{|l|}{ R.m.s. deviations } \\
\hline Bond lengths ( $\AA$ ) & 0.017 & 0.008 & 0.017 \\
\hline Bond angles $\left({ }^{\circ}\right)$ & 1.713 & 1.132 & 1.675 \\
\hline PDB code & $4 w 4 q$ & 3wxs & $3 w x q$ \\
\hline
\end{tabular}

